\title{
A Rare Case of Type I Renal Tubular Acidosis with Membranous Nephropathy Presenting as Hypokalemic Paralysis
}

\author{
Sham Sunder ${ }^{\mathrm{a}} \quad$ Satyanand Sathi $^{\mathrm{a}} \quad$ K. Venkataramanan ${ }^{\mathrm{a}}$ \\ Himanshu Verma ${ }^{a}$ Minakshi Bhardwaj $^{\mathrm{b}} \quad$ J. Rajesh $^{\mathrm{a}}$ \\ Himanshu Mahapatra ${ }^{a}$ \\ Departments of ${ }^{a}$ Nephrology and ${ }^{b}$ Pathology, PGIMER, Dr. R.M.L. Hospital, \\ New Delhi, India
}

\section{Key Words}

Membranous nephropathy · Distal renal tubular acidosis · Medullary nephrocalcinosis . Hypokalemic paralysis

\begin{abstract}
Type 1 renal tubular acidosis (RTA), or distal RTA (dRTA), is a disorder of renal tubular acidification, which is generally asymptomatic but may rarely present as hypokalemic paralysis. Here, we report the case of a young male who presented with sudden onset weakness of all 4 limbs and a 2-month history of swelling of the legs. An investigation revealed hypokalemia, metabolic acidosis, and nephrotic syndrome. Additional analyses revealed normal anion gap metabolic acidosis with a positive urine anion gap and dRTA. Renal biopsy showed evidence of membranous nephropathy (MN). The patient's weakness improved with potassium supplements. Normalization of the serum potassium level and disappearance of proteinuria were established with an ACE inhibitor and potassium supplementation. This case is an unusual combination of dRTA with MN coupled with the rare presenting symptoms of hypokalemic paralysis and medullary nephrocalcinosis.
\end{abstract}




\section{Introduction}

Type 1 renal tubular acidosis (RTA), or distal RTA (dRTA), is a disorder of renal tubular acidification, characterized by hyperchloremic metabolic acidosis with a normal serum anion gap and a persistently high urinary $\mathrm{pH}$. Hypokalemia has been reported in $28-53 \%$ of patients with type 1 RTA [1]. dRTA is generally asymptomatic but rarely may present as hypokalemic paralysis. Other than the hereditary and idiopathic varieties, most cases of dRTA are secondary to systemic disorders such as Sjögren's syndrome, hyperglobulinemia, chronic active hepatitis, or lupus. In nondiabetic adults, membranous nephropathy (MN) is the most common cause of nephrotic syndrome, accounting for up to one-third of biopsy diagnoses. MN in adults is most often idiopathic (approximately 75\% of cases). About onethird of cases are secondary to infection, rheumatologic disorders, neoplasm, and exposure to drugs and toxins. Here, we report a hitherto unusual combination of dRTA with MN presenting as hypokalemic paralysis and medullary nephrocalcinosis.

\section{Case Report}

A 20-year-old male presented with a 2-month history of on and off swelling of both legs and sudden onset weakness of all 4 limbs without any history of loss of consciousness, seizure, headache, sensory loss, or bowel and bladder involvement. There was no history of such a weakness. Additionally, there was no history of dryness of the mouth and eyes (suggestive of Sjögren's syndrome), no joint pain, oral ulcers, hair loss, or photosensitivity (suggestive of lupus). A neurological examination revealed normal cranial nerves and higher mental functions. Power in the upper and lower limbs was $2 / 5$ and $1 / 5$, respectively. His deep tendon reflexes were diminished, with no plantar response; however, sensory function remained intact.

The patient's laboratory profile was as follows: hemoglobin: $10.8 \mathrm{~g} / \mathrm{dl}$, total leukocyte count: $14,300 / \mathrm{mm}^{3}$, platelet count: $2.8 \times 10^{5} / \mathrm{mm}^{3}$, urinary protein: $3+$, urinary sugar: 0 , urine microscopy: white blood cell count: $4-6 /$ high-power field, red blood cell count: $2-$ 4/high-power field, urinary $\mathrm{pH}$ : -6.5, 24-hour urinary protein: $3.3 \mathrm{~g} /$ day, serum albumin: 2.1 $\mathrm{g} / \mathrm{dl}$, serum cholesterol: $526 \mathrm{mg} / \mathrm{dl}$, serum triglycerides: $346 \mathrm{mg} / \mathrm{dl}, \mathrm{C} 3: 79.6 \mathrm{mg} / \mathrm{dl}$ (normal range: $80-160$ ), C4: $62.8 \mathrm{mg} / \mathrm{dl}$ (normal range: 20-40), HBsAg: negative, anti-HCV: negative, HIV I and II: negative, anti-Ro and anti-La antibodies: negative, blood urea: $29 \mathrm{mg} / \mathrm{dl}$, serum creatinine: $1.2 \mathrm{mg} / \mathrm{dl}$, random blood sugar: $88 \mathrm{mg} / \mathrm{dl}$, corrected serum calcium: $9.4 \mathrm{mg} / \mathrm{dl}$, serum sodium: $143 \mathrm{mEq} / \mathrm{l}$, serum potassium: $2.0 \mathrm{mEq} / \mathrm{l}$, serum chloride: $120 \mathrm{mEq} / \mathrm{l}$, serum $\mathrm{PO}_{4}: 4.3 \mathrm{mEq} / \mathrm{l}$, arterial blood gas: $\mathrm{pH} 7.2, \mathrm{pCO}_{2}: 31 \mathrm{~mm} \mathrm{Hg}, \mathrm{pO}_{2}: 104 \mathrm{~mm} \mathrm{Hg}, \mathrm{HCO}_{3}: 12.5$ $\mathrm{mEq} / \mathrm{l}$, anion gap: $10.5 \mathrm{mEq} / \mathrm{l}$ (normal range: $10-12$ ).

The urine anion gap (UAG) $\left[\mathrm{Na}^{+}+\mathrm{K}^{+}-\mathrm{Cl}^{-}(70+25-65=30)\right]$ was positive. Urinary $\mathrm{pH}$ was $<5.5$ and the fractional excretion of bicarbonate $\left(\mathrm{FeHCO}_{3}\right)$ was found to be 2, after intravenous infusion of sodium bicarbonate. Thus, the diagnosis of dRTA was established. Ultrasonography showed normal-sized kidneys with nephrocalcinosis of the medulla (suggestive of type $1 \mathrm{RTA}$ ), mild right-sided pleural effusion, and moderate ascites. A renal biopsy showed a thickened basement membrane with subepithelial spikes (fig. 1, fig. 2). The interstitium showed mild mononuclear cell infiltrate and fibrosis with tubular involvement (fig. 3). Immunofluorescence was positive for IgG and C3 (fig. 4). Thus, the diagnosis of MN was made. 


\section{Treatment}

For hypokalemic paralysis, the patient was initially treated with $60 \mathrm{mEq}$ potassium chloride (KCl) orally, followed by $30 \mathrm{mEq} \mathrm{KCl}$ orally every 30 min until serum potassium had normalized. After potassium supplementation, the patient's weakness improved within 2 days and he was able to walk again. Repeat investigations showed serum potassium: 4.8 $\mathrm{mEq} / \mathrm{l}$ (normal), serum chloride: $112 \mathrm{mEq} / \mathrm{l}$, arterial blood gas: $\mathrm{pH} 7.25, \mathrm{HCO}_{3}: 15.7 \mathrm{mEq} / \mathrm{l}$, anion gap: $11.3 \mathrm{mEq} / \mathrm{l}$ (normal 10-12). Due to proteinuria and hypokalemia, $5 \mathrm{mg}$ enalapril b.d. was started. After $24 \mathrm{~h}$, proteinuria was less than $4 \mathrm{~g} /$ day and a renal function test was found to be normal; immunosuppressive therapy was not initiated, as per KDIGO guidelines [2]. The patient responded well to treatment with $5 \mathrm{mg}$ enalapril b.d. and $10 \mathrm{ml}$ potassium citrate t.d.s. Normal potassium levels and no proteinuria have been observed to date (24 months of follow-up). ANA (antinuclear antibody), dsDNA, anti-Ro, and anti-La antibody tests were done to establish the etiology, but were found to be negative. Schirmer's test was also negative (more than $10 \mathrm{~mm}$ of moisture on the filter paper in $5 \mathrm{~min}$ was noted).

\section{Discussion}

Hypokalemic paralysis is primarily the result of either hypokalemic periodic paralysis (HPP) caused by an enhanced shift of potassium into cells or secondary HPP resulting from excessive gastrointestinal or urinary potassium loss. Secondary HPP requires higher doses of $\mathrm{KCl}$ [3]. Our patient required a large dose of $\mathrm{KCl}$ for the correction of hypokalemic paralysis. In the presence of hypokalemia, urinary potassium $<20 \mathrm{mmol} /$ day suggests extra renal loss, whereas urinary potassium $>20 \mathrm{mmol} /$ day suggests renal loss, as was the case in our patient. The UAG represents an indirect index of urinary $\mathrm{NH} 4+$ excretion in patients with hyperchloremic metabolic acidosis [4]. The UAG is defined by the equation: $\mathrm{UAG}=\mathrm{Na}^{+}+\mathrm{K}^{+}-$ $\mathrm{Cl}^{-}$[5]. In our patient, the UAG was positive $(30 \mathrm{mmol} / \mathrm{l})$, suggestive of renal loss of potassium.

Though RTA is a tubulointerstitial kidney disease, it may also be seen in primary glomerulopathies. Primary glomerulopathies may cause damage to tubules and the interstitium with functional derangement. In glomerular diseases associated with proteinuria, tubular cells may be activated by elevated levels of normal and novel urinary proteins. Activated tubular cells may synthesize proinflammatory mediators, especially monocyte chemoattractant molecules (e.g. MCP-1, RANTES, complement component, and fractalkine) and fibrosis-promoting molecules (e.g. endothelin, TGF- $\beta$, and angiotensin II). Damage to the tubular basement membrane facilitates the passage of tubular-derived products into the interstitium and peritubular capillaries spaces. In the distal nephron, protein casts may obstruct urinary flow and aggravate tubulointerstitial damage [6]. Glomerulus-derived cytokines activating tubular epithelial cells reduce peritubular blood flow, leading to tubulointerstitial ischemia and hyperfunction of remnant tubules. Peritubular ischemia contributes more to tubular damage than proteinuria in immune-mediated glomerulonephritis [7]. Occasionally, the same inflammatory mechanism of glomerulonephritis may also affect the tubules. For example, dRTA occurring in immunological disorders such as Sjögren's syndrome and systemic lupus erythematosus is thought to result from an interstitial nephritis which various investigators have ascribed to mononuclear cell infiltration, deposition of antigen-antibody complexes along tubular basement membranes, and the formation of antibodies directed against the tubular basement membrane. A literature search revealed cases in which features of dRTA preceded other manifestations of 
Sunder et al.: A Rare Case of Type I Renal Tubular Acidosis with Membranous Nephropathy Presenting as Hypokalemic Paralysis

systemic lupus erythematosus by 4-6 years [8]. In our patient, lupus and Sjögren's syndrome were ruled out. A case of dRTA with MN has been reported [9]. HPP due to proximal RTA with membranoproliferative glomerulonephritis has also been reported [10]. But dRTA with MN presenting as hypokalemic paralysis has not been reported. Long-term correction of the hypokalemia in our patient was important not only to overcome the neuromuscular impairment, but also to prevent further deterioration of kidney function. It has been suggested that hypokalemia stimulates interstitial deposition of complement components ( $\mathrm{C} 3$ and $\mathrm{C} 5 \mathrm{ba}$ ), which is conducive to the aggravation of renal interstitial damage [11].

About $30-35 \%$ of patients with idiopathic MN eventually undergo spontaneous remission of nephrotic syndrome; therefore, it is reasonable to delay specific immunosuppressive therapy for at least 6 months, instead utilizing supportive therapy including RAS blockade unless the patient has unexplained rapid deterioration in kidney function or there are complications related to uncontrolled nephrotic syndrome. Our patient was treated with RAS blockade and responded well. KDIGO recommend that initial immunosuppressive therapy be started only in patients with nephrotic syndrome and when at least one of the following conditions is met: urinary protein excretion persistently exceeds $4 \mathrm{~g} / \mathrm{d}$ and remains at over $50 \%$ of the baseline value, and/or it does not show progressive decline despite antihypertensive and antiproteinuric therapy during an observation period of at least 6 months (1B; level 1: recommend; B: moderate quality of evidence) [2].

dRTA is a clinical syndrome consisting of hypokalemia, hyperchloremic metabolic acidosis, inability to lower urinary pH below 5.5 during acidemia nephrolithiasis, and nephrocalcinosis. Hypokalemia is frequently seen in dRTA [12] and may sometimes be severe enough to produce muscle weakness, as in our case. Both hypercalciuria and especially hypocitraturia contribute to the development of nephrocalcinosis and nephrolithiasis [13]. It is very evident that our patient had dRTA (type 1 RTA) with medullary nephrocalcinosis. Correction of acidosis with alkali therapy (usually potassium citrate) generally improves both calcium and potassium balance, and prevents stones and nephrocalcinosis. In contrast to the high alkali requirement in proximal RTA, only daily acid load $(1-2 \mathrm{mEq} / \mathrm{kg} /$ day $)$ has to be replenished with alkali therapy in dRTA.

\section{Conclusion}

Primary glomerulopathies may cause tubulointerstitial damage, which may subsequently lead to the development of RTA. RTA may be the first manifestation of glomerulopathy, preceding other clinical features. We have reported the case of an unusual association of dRTA with MN, with the rare presentation of hypokalemic paralysis, which was successfully treated.

\section{References}

1 Hattori N, Hino M, Ishihara T, et al: Hypokalemic paralysis associated with distal renal tubular acidosis. Intern Med 1992;31:662-665.

2 Kidney Disease: Improving Global Outcomes (KDIGO) glomerulonephritis work group: KDIGO clinical practice guideline for glomerulonephritis. Kidney Int Suppl 2012;2:139-274.

-3 Lin SH, Chiu JS, Hsu CW, Chau T: A simple and rapid approach to hypokalemic paralysis. Am J Emerg Med 2003;21:487-491.

4 Rodriguez Soriano J: Renal tubular acidosis: the clinical entity. J Am Soc Nephrol 2002;13:2160-2170.

5 Penny MD, Oleesky DA: Renal tubular acidosis. Ann Clin Biochem 1999;36:408-422. 
Sunder et al.: A Rare Case of Type I Renal Tubular Acidosis with Membranous Nephropathy Presenting as Hypokalemic Paralysis

-6 Segerer S, Nelson PJ, Schlondorff D: Chemokines, chemokine receptors, and renal disease: from basic science to pathophysiologic and therapeutic studies. J Am Soc Nephrol 2000;11:152-176.

-7 Wong MG, Suzuki Y, Tanifuji C, et al: Peritubular ischemia contributes more to tubular damage than proteinuria immune-mediated glomerulonephritis. J Am Soc Nephrol 2008;19:290-297.

-8 Ter Meulen CG, Pieters GFFM, Huysmans FTM: Flaccid paresis due to distal renal tubular acidosis preceding systemic lupus erythematosus. Neth J Med 2002;60:29-32.

9 Padamnabhan S, Lakshmi AY, ShivaKumar V: Membranous nephropathy and distal RTA with medullary nephrocalcinosis. Indian J Nephrol 2005;15:33-34.

10 Santra G, De D, Sinha PK: Hypokalemic periodic paralysis due to proximal renal tubular acidosis in a case with membranoproliferative glomerulonephritis. J Assoc Physicians India 2011;59:735-737.

11 Nakhoul F, Plavnic Y, Lichtig H, Better OS: Hypokalemic flaccid paralysis as the presenting symptom of autoimmune interstitial nephropathy. Isr J Med Sci 1993;29:300-303.

-12 Batlle D, Moorthi KM, Schlueter W, Kurtzman N: Distal renal tubular acidosis and the potassium enigma. Semin Nephrol 2006;26:471.

-13 Brenner RJ, Spring DB, Sebastian A, et al: Incidence of radiographically evident bone disease, nephrocalcinosis, and nephrolithiasis in various types of renal tubular acidosis. N Engl J Med 1982;307:217.

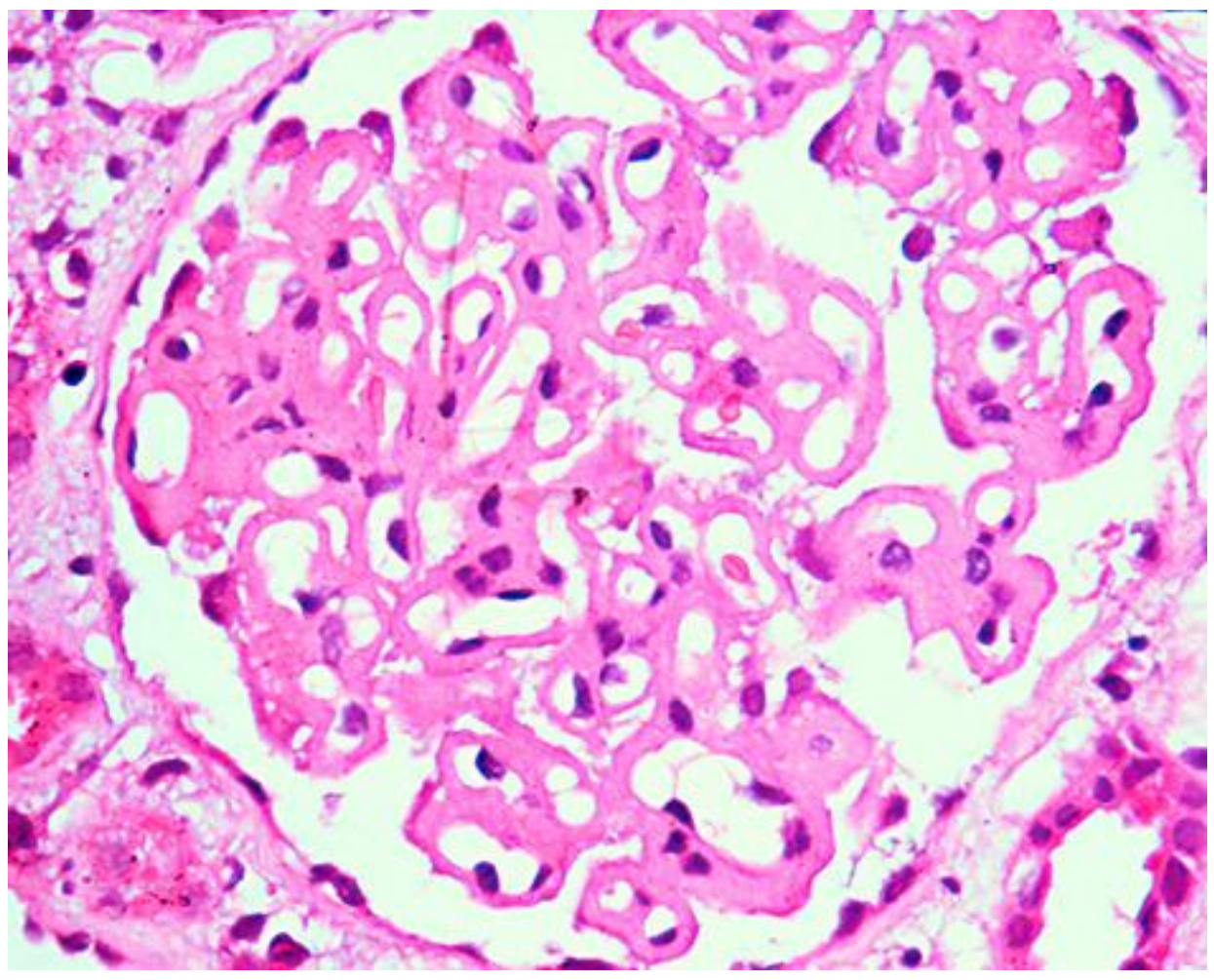

Fig. 1. Kidney biopsy specimen shows a uniform increase in thickness of the glomerular capillary wall. 
Case Reports in

Nephrology and
Case Rep Nephrol Urol 2013;3:91-98

DOI: $10.1159 / 000353768$

Sunder et al.: A Rare Case of Type I Renal Tubular Acidosis with Membranous

Nephropathy Presenting as Hypokalemic Paralysis

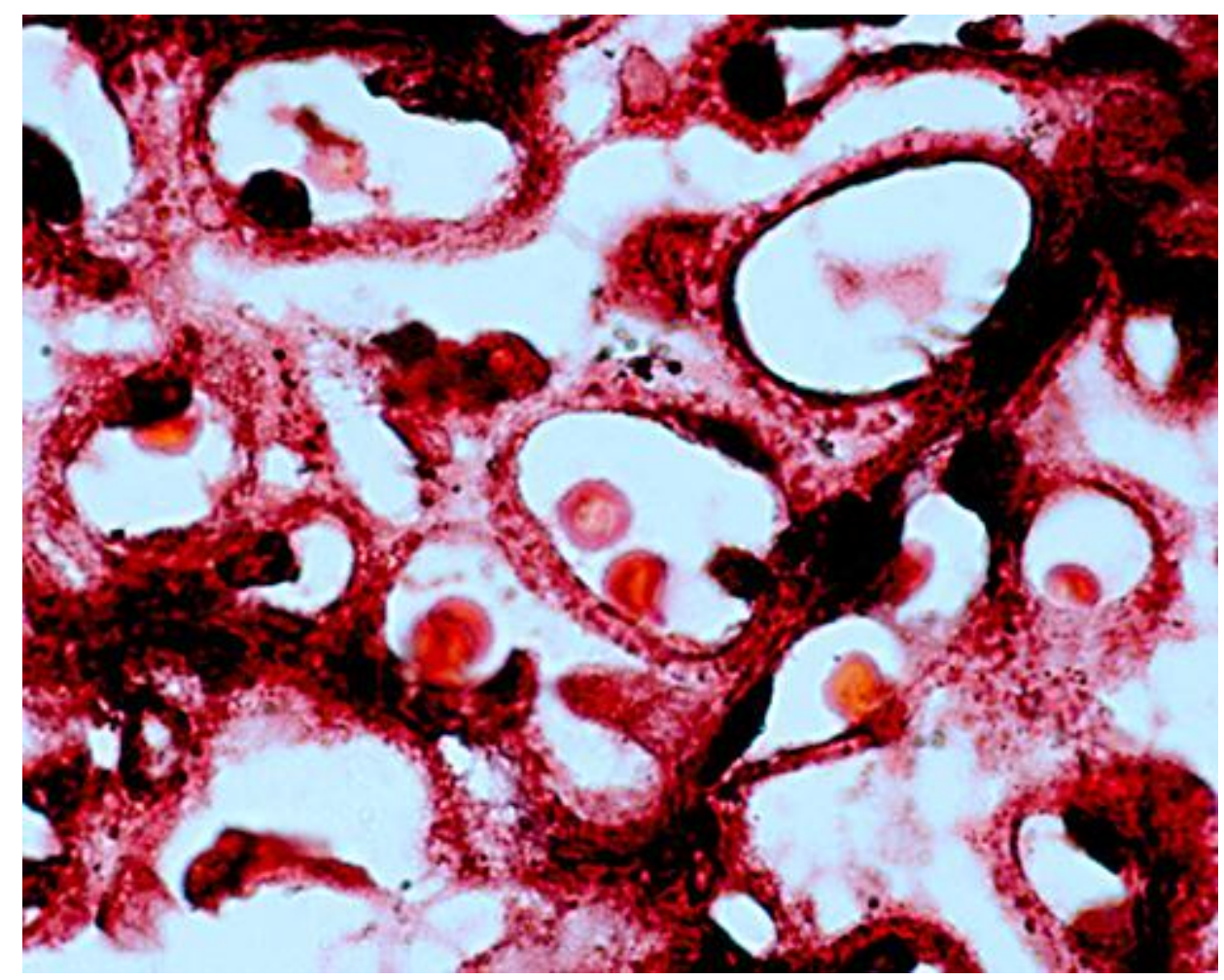

Fig. 2. Kidney biopsy specimen shows a thickened basement membrane with subepithelial spikes, diagnostic of MN. Methenamine silver stain. 


\section{Case Reports in \\ Nephrology and \\ Urology}

\begin{tabular}{l|l}
\hline Case Rep Nephrol Urol 2013;3:91-98 \\
\hline DOI: 10.1159/000353768 & $\begin{array}{l}\text { @ 2013 S. Karger AG, Basel } \\
\text { www.karger.com/cru }\end{array}$ \\
\hline
\end{tabular}

Sunder et al.: A Rare Case of Type I Renal Tubular Acidosis with Membranous

Nephropathy Presenting as Hypokalemic Paralysis

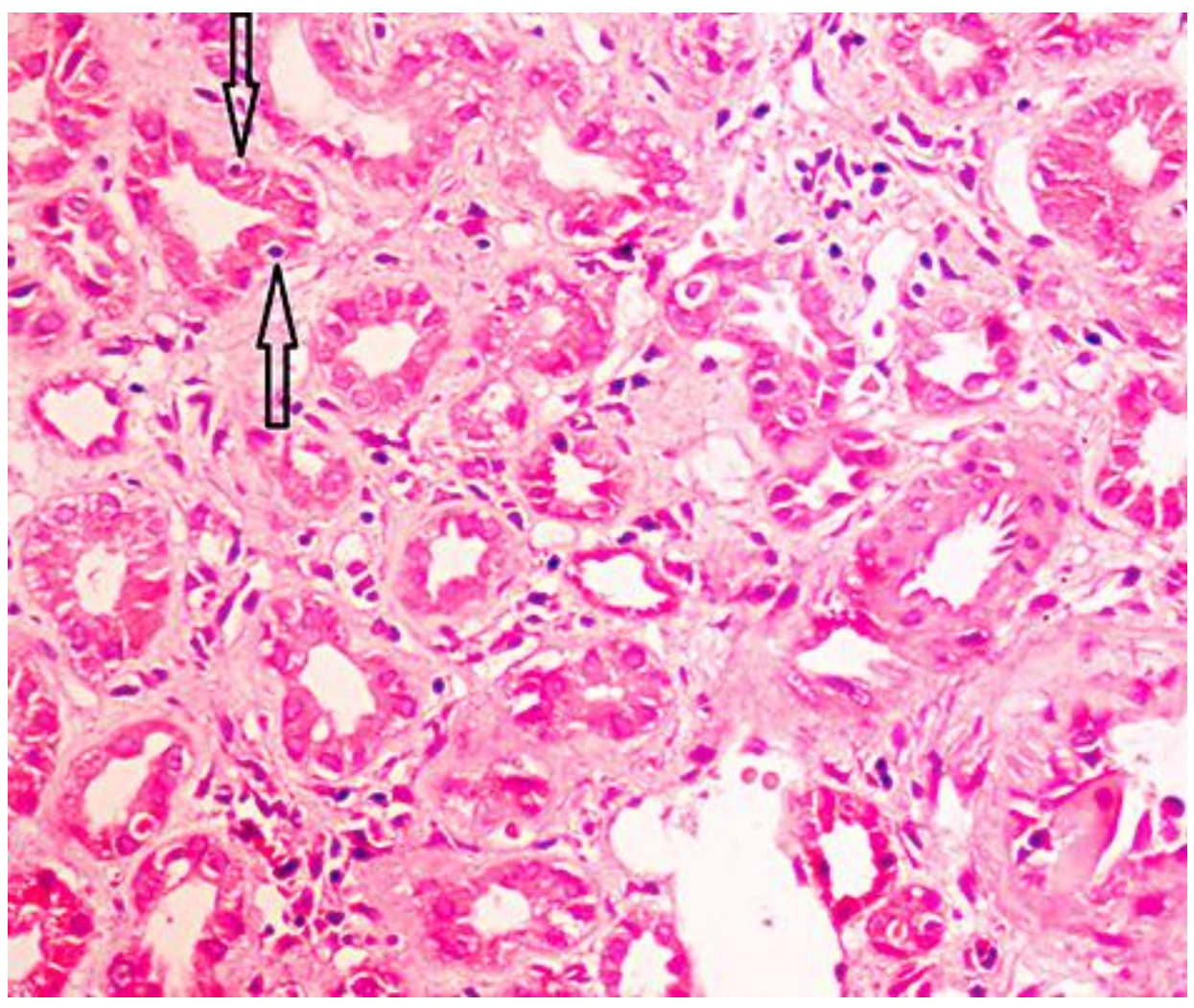

Fig. 3. Diffuse tubulointerstitial mononuclear infiltrate (arrows show mononuclear cells infiltrating the base of the tubule) and mild fibrosis. 
Case Reports in

Nephrology and

Urology
Case Rep Nephrol Urol 2013;3:91-98

DOI: $10.1159 / 000353768$

Sunder et al.: A Rare Case of Type I Renal Tubular Acidosis with Membranous

Nephropathy Presenting as Hypokalemic Paralysis

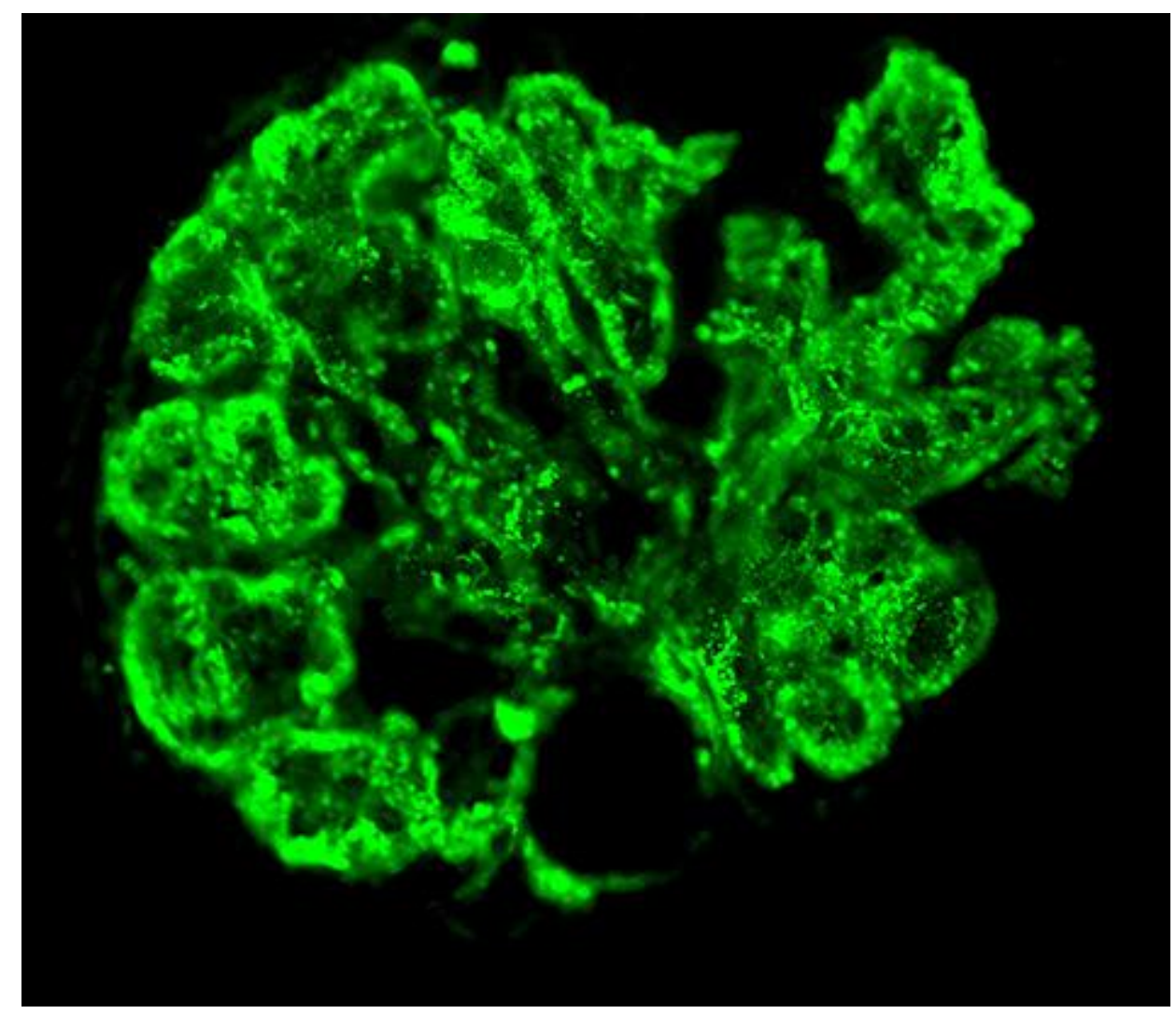

Fig. 4. Immunofluorescence shows $\operatorname{IgG}$ positivity and fine, granular deposition of IgG along the outer surface of the capillary walls. 